\title{
Comment on Migliorini et al.: short stems for total hip replacement among middle-aged patients
}

\author{
Shunyu Yao ${ }^{1} \cdot$ Kai Zhou $^{1} \cdot$ Zongke Zhou $^{1}$ \\ Received: 23 March 2020 / Accepted: 31 March 2020 / Published online: 12 April 2020 \\ (C) SICOT aisbl 2020
}

Dear Editor,

We read with great interest the article entitled "Short stems for total hip replacement among middle-aged patients" by Migliorini et al. [1]. The authors performed an interesting meta-analysis to compare outcomes of short stems implants and conventional implants of middle-aged patients undergoing total hip arthroplasty (THA). However, the meta-analysis has brought several questions to our minds that we would like to communicate with the authors.

First, the authors mentioned that a variety of databases were used in this study, but they were not enough. It would make the outcomes more convincing if the authors included other databases, like clinicaltrials.gov, MEDLINE, NLM Gateway, and BIOSIS previews, to obtain more literature. Besides, on the 14 studies included, only five of them performed randomization and avoid selection bias, which means almost two-thirds of them have selection bias. In those study, short stem implants were used in selected patients. Combined with the ratio in included studies, the substrate to start this meta-analysis was weaker.

Second, in the result of the article, the author said the short stem group showed a statistically significant greater value of the WOMAC score compared with the standard stem group (MD, 7.92; 95\% CI, 3.21 to 12.63; $p=0.001$; Fig. 4). But Fig. 4 shows no significant difference (MD, $-0.08 ; 95 \% \mathrm{Cl}$ $-1.58,1.43 ; p=0.92$ ). We considered the title of Fig. 4 and Fig. 5 is reversed. Figure 4 should be forest plot of the comparison Harris hip score and Fig. 5 should be forest plot of the WOMAC score comparison. Also, in Fig. 5, one single-study weight $85.7 \%$ is significantly higher than others, though no high heterogeneity was found; more study should be included in meta-analysis. With heterogeneities found in this WOMAC score $\left(I^{2}=39 \%\right)$, subgroup analysis and sensitivity analysis had been conducted to explain the source of heterogeneity.

Third, the PICO algorithm of the study mentioned population of middle-aged 40 to 69 years, but this study only concerns with a mean age from 45 to 69 . Some study with mean age in 40 to 45 should be included. There is no clear definition of middle-aged, but ages 65 to 69 may not be included.

Finally, it would be better if the publication biases of all included studies were assessed by funnel plots, Begg's test, and Egger's test. In the meta-analysis, two included studies were carried out in Asian and others were in Caucasian; therefore, the ethnic bias should be considered. We appreciate that Filippo Migliorini et al. provided us with an interesting metaanalysis focusing to compare outcomes of short stem implants and conventional implants of middle-aged patients undergoing THA. However, more studies based on scientifically designed RCTs with long-term follow-up and large samples should be carried out to clear these issues.

\section{Reference}

1. Migliorini F, Driessen A, Colarossi G et al (2020 Mar 19) Short stems for total hip replacement among middle-aged patients. Int Orthop. https://doi.org/10.1007/s00264-020-04516-x

Publisher's note Springer Nature remains neutral with regard to jurisdictional claims in published maps and institutional affiliations.

Zongke Zhou

zhouzongke@scu.edu.cn

1 Department of Orthopaedics, West China Hospital of Sichuan University, Chengdu 610041, China 